\title{
Effects of Farinelli Breating Exercise on Respiratory Function and Symptoms in Patients with Chronic Obstructive Pulmonary Disease
}

\author{
Supawit Ittinirundorn, M.Sc. ${ }^{1,2}{ }^{\circledR}$, Naiyana Wongsaita, Ph.D, R.N. ${ }^{3}$, Dujrath Somboonviboon, M.D. ${ }^{4}$ \\ and Wannaporn Tongtako, Ph.D. ${ }^{1,2}$ [D \\ ${ }^{1}$ Exercise Physiology in Special Population Research Unit, Chulalongkorn University, Bangkok, ${ }^{2}$ Area of Exercise Physiology, \\ Faculty of Sports Science, Chulalongkorn University, Bangkok, ${ }^{3}$ Royal Thai Army Nursing College, Bangkok, ${ }^{4}$ Pulmonary and \\ Critical Care Division, Department of Medicine, Phramongkutklao Hospital, Bangkok, Thailand
}

Background: Farinelli breathing (FB) exercise is a typical breathing exercise used by singers. This study aimed to compare effects of FB exercise and diaphragmatic breathing (DB) exercise on respiratory function and symptoms in patients with chronic obstructive pulmonary disease (COPD).

Methods: Sixteen patients aged 51-80 years with mild or moderate COPD were recruited for this study. They were divided into two groups: DB group $(n=8)$ and FB group $(n=8)$. Both groups received complete breathing exercise training five times per week for 8 weeks. Their respiratory functions, COPD symptoms, cytokine levels, and oxidative stress variables were analyzed during pre- and post-tests. Dependent variables were compared between pre- and posttests using paired t-tests. An independent t-test was used to compare variables between the groups. Differences were considered significant at $\mathrm{p}<0.05$.

Results: The maximal expiratory pressure (MEP), maximum oxygen consumption ( $\mathrm{VO}_{2} \mathrm{max}$ ), and COPD Assessment Test (CAT) scores changed significantly in the DB group after the 8-week intervention compared to those at pre-test, whereas force vital capacity, forced expiratory volume in the first second, maximum voluntary ventilation, maximal inspiratory pressure (MIP), MEP, $\mathrm{VO}_{2} \mathrm{max}$, CAT score, tumor necrosis factor- $\alpha$, and malondialdehyde level changed significantly in the FB group at post-test compared to those at pre-test. Moreover, both MIP and MEP in the FB group were significantly higher than those in the DB group.

Conclusion: FB exercise improved respiratory functions and COPD symptoms of patients with COPD. It might be an alternative breathing exercise in pulmonary rehabilitation programs for patients with COPD.

Keywords: Farinelli Breathing; Chronic Obstructive Pulmonary Disease Patients; Respiratory Function; COPD Questionnaire Scores

Address for correspondence: Wannaporn Tongtako, Ph.D.

Faculty of Sports Science, Chulalongkorn University, Rama I Rd., Wangmai, Pathumwan District, Bangkok 10330, Thailand

Phone: 662-218-1035, Fax: 662-218-1420, E-mail:wannaporn.t@chula.ac.th

Received: Jul. 21, 2021, Revised: Oct. 20, 2021, Accepted: Dec. 27, 2021, Published online: Dec. 29, 2021

@(c) it is identical to the Creative Commons Attribution Non-Commercial License (http://creativecommons.org/licenses/by-nc/4.0/) 


\section{Introduction}

Chronic obstructive pulmonary disease (COPD) is the third leading cause of death globally. COPD is a common, treatable, and preventable disease characterized by airflow limitation and persistent respiratory system infections resulting from exposure to noxious gases and particles ${ }^{1,2}$. Airflow limitation caused by irreversible airway restriction and emphysema is a hallmark of COPD. It can lead to a decrease in maximum oxygen consumption ${ }^{3}$. Chronic cough, sputum production, and dyspnea are the most important symptoms in patients with COPD. The mechanism of COPD can be explained by decreased oxygen with increased hydrogen ions $\left(\mathrm{H}^{+}\right)$and carbon dioxide in the aorta, leading to a sensation of respiratory discomfort and a feeling of breathlessness. At this point, the medulla oblongata fires respiratory drive signals to receptors in the lungs and respiratory muscles, resulting in breathing ${ }^{4}$. Thus, breathing exercises can improve gas exchange, decrease gas trapping, and alter the breathing pattern ${ }^{5-7}$. Patients with COPD use their accessory respiratory muscles due to air trapping (hyperinflation), abnormal thoracic movement, and sarcomere shortening of the diaphragm, which can result in labored breathing that impairs their daily activities and exercise tolerance $^{8,9}$. Pathophysiological changes in the pulmonary tissue, airways, and pulmonary blood vessels are associated with inflammatory cytokines and oxidative stress in patients with $\mathrm{COPD}^{10,11}$. Reactive oxygen species (ROS) and reactive nitrogen species production initiated by inflammatory processes and mitochondrial respiration can result in membrane and DNA damage ${ }^{12,13}$. Moreover, the release of inflammatory cytokines such as tumor necrosis factor- $\alpha$ (TNF- $\alpha$ ) and interleukin-6 in patients with prolonged inflammation is associated with disease progression ${ }^{11,14}$.

Breathing exercises such as low-intensity aerobic exercise can improve exercise tolerance, increase respiratory muscle strength and endurance, correct abnormal chest and abdominal movements, and diminish hyperinflation ${ }^{15-18}$. Several types of breathing exercises such as deep breathing, pursed-lip breathing, and diaphragmatic breathing (DB) are fundamental parts of comprehensive pulmonary rehabilitation programs for patients with $\mathrm{COPD}{ }^{18,19}$. Zhang et $\mathrm{al}^{20}{ }^{20}$ have studied effects of conventional breathing exercises in patients with COPD. For 8 weeks, patients practiced inhaling quickly ( 0.8 to 1 second), holding their breath, and exhaling slowly (3 to 4 seconds). They found that their maximal inspiratory muscle pressure (MIP), maximal expiratory pressure (MEP), and 6-minute walk distance (6-MWD) were increased. Moreover, a previous study has demonstrated that 4 weeks of incentive spirometry in patients with COPD not only increased their pulmonary function and respiratory muscle strength, but also reduced their inflammatory cytokines and oxidative stress ${ }^{11}$. In singers, benefits of breathing exercises include breath control with improved respiratory muscle strength, which can improve vocal efficiency ${ }^{21}$. Farinelli breathing (FB) exercise is a traditional breathing exercise in singers that consists of three phases: inhalation, suspension, and expiration. The principle of this breathing exercise involved inhaling slowly through the nasal cavity with the activation of the diaphragm and exhaling slowly with a pursed lip. Interestingly, previous studies have shown that singers have better pulmonary functions than non-singers ${ }^{22,23}$. To the best of our knowledge, this is the first study to apply singers' breathing exercises in patients with COPD. Furthermore, effects of these FB and DB exercises on respiratory function and symptoms in patients with COPD were compared to determine whether FB should be considered in pulmonary rehabilitation for patients with COPD. DB is a classic breathing exercise with the goal to improve gas exchange and chest expansion. Previous studies have indicated that DB can improve lung volumes, respiratory motion, oxygen saturation $\left(\mathrm{SpO}_{2}\right)$, respiratory rate, and sensation of dyspnea and reduce fatigue during exercise in patients with $\mathrm{COPD}^{24-26}$. Thus, the objective of this study was to compare effects of FB and DB exercises on pulmonary function, respiratory muscle strength, aerobic capacity, COPD symptoms, cytokine levels, and oxidative stress in patients with COPD.

\section{Materials and Methods}

\section{Study design and subjects}

This study utilized a pre-test/post-test interventional research design. It was approved by the Institutional Review Board of the Royal Thai Army Medical Department, Thailand (Study Code: IRBRTA 1347/2562). This study was registered as a clinical trial with clinical trials.gov (no. NCT04869033). The sample size of participants was calculated using the $\mathrm{G}^{*}$ Power program (3.1.9.2) with an alpha error of 0.05 and a power of 0.80. A minimum of six participants in each group were required for this study. Nineteen patients with COPD were recruited from Phramongkutklao Hospital for this study. All participants gave written informed consent prior to participation in the study. These patients were assigned to FB and DB groups using random allocation by investigators and stratified by sex (male and female), age (51-60, 61-70, and 71-80 years old), and COPD severity (mild and moderate). The severity was determined after repeated pulmonary function tests based on the predicted forced expiratory volume in the first second $\left(\mathrm{FEV}_{1} \%\right)$ and $\mathrm{FEV}_{1}$ / force vital capacity $(\mathrm{FVC})$ ratio according to the 2021 Global Initiative for Chronic Obstructive Lung Disease. Inclusion criteria were as follows: (1) patients with COPD who were treated at Phramongkutklao Hospital; (2) having a history of smoking; (3) predicted $\mathrm{FEV}_{1}$ of more than $50 \%$ after bronchodilator; (4) no change in medication whitin 4 weeks preceding the study; (5) no history of acute exacerbations within 4 weeks befor this study; and (6) no history 
of cardiac disease. Exclusion criteria were as follows: (1) recurrent acute exacerbations; (2) inability to participate in at least $80 \%$ of the training program ( $\leq 32$ sessions of 40 sessions); (3) unwillingness to continue performing the exercise.

Participants were contacted. They underwent a 2-hour orientation, which included assessment training, after their main healthcare practitioner granted them medical clearance to participate (after blood collecting, physiological characteristics, pulmonary function, respiratory muscle strength, 6-MWD, and COPD questionnaires) in the intervention procedures (breathing exercises). Data were collected in a confidentiality and privacy room of the hospital. Health history, COPD questionnaires (including the modified Medical Research Council Dyspnea Scale [mMRC], and COPD Assessment Test [CAT] were obtained or performed through interviews on the preexperimental day. Characteristics such as age, weight, body mass index (BMI), blood pressure, percentage of body fat, and $\mathrm{SpO}_{2}$ were recorded. Blood was collected into sterile ethylenediaminetetraacetic acid (EDTA) tubes from the cubital vein by a nurse at Phramongkutklao Hospital and analyzed by a medical technologist. EDTA blood was centrifuged at 3,000 rpm for 10 minutes to obtain plasma. All plasma samples were stored at $-80^{\circ} \mathrm{C}$ before their use in different assays. Pulmonary function was assessed repeatedly before evaluating respiratory muscle strength and the 6-MWD. All participants were randomly assigned to the DB or FB group. They continued to receive their regular medication.

Following the initial examination, participants were given a breathing exercise manual notebook that contained program instructions and an activity diary to evaluate program adherence. Each participant was given a personalized home breathing exercise regimen ( 5 days per week for 8 weeks), which was closely monitored by researchers. Participants were monitored during breathing exercise sessions. They continued to take their prescribed bronchodilator medications. Furthermore, researchers called all participants twice a week to correct the performance of the breathing exercise as needed, to inquire about any barriers that participants encountered during sessions, and to verify the correct performance of the breathing exercise.

\section{FB and DB exercise programs}

This study was designed with two different types of breathing exercises: DB and FB. These groups were differentiated by the route and time in seconds of inhalation, suspension, and exhalation. Both groups were instructed to practice in a seated position on a chair with back support, starting with normal breathing (NB) for 1 minute, followed by a different breathing pattern. In the DB group, patients inhaled through the nose and exhaled through the mouth. After 1 minute of NB, patients inhaled for 2 seconds and exhaled for 2 seconds through the nasal airway. This pattern was continued for 4 minutes.
Patients then returned to NB for 1 minute. One minute of NB and 4 minutes of DB were considered as one set. Participants were asked to perform six sets per day, 5 days per week (Monday-Friday) for weeks $1-4$. For weeks $5-8$, they were aksed to perform eight sets per day, 5 days per week.

In the FB group, patients inhaled through the nose, held their breath, and exhaled using pursed lips. After 1 minute of NB, patients followed the following set of instructions: (1) inhaled for 2 seconds, suspended for 2 seconds, exhaled for 2 seconds; (2) inhaled for 3 seconds, suspended for 3 seconds, exhaled for 3 seconds; (3) inhaled for 4 seconds, suspended for 4 seconds, exhaled for 4 seconds; (4) inhaled for 5 seconds, suspended for 5 seconds, exhaled for 5 seconds; and (5) inhaled for 6 seconds, suspended for 6 seconds, exhaled for 6 seconds. This cycle lasted for 1 minute. It was repeated a total of four times. One minute of NB and 4 minutes of FB were considered as one set. Participants were asked to perform six sets per day, 5 days per week (Monday-Friday) for weeks $1-4$. For weeks 5-8, they were asked to perform eight sets per day, 5 days per week.

\section{Pulmonary function test}

Pulmonary function and breathing pattern were assessed using the predicted value and liters of FVC, vital capacity (VC), and the maximum voluntary ventilation (MVV) maneuver analyzed with a computerized spirometer (MIR Spirobank G, Rome, Italy) according to guidelines of the American Thoracic Society pulmonary function test. Participants were asked to wear a nose clip while sitting on a chair. The researcher gave participants a step-by-step protocol to prevent incorrect maneuvers. For FVC maneuver, three cycles of slow NB were performed before demonstrating forced inspiration and expiration and returning to NB. For the VC maneuver, NB inhalation and exhalation were performed until the signal of the device sounded, followed by deep and slow inhalation and exhalation. For the MVV maneuver, participants were asked to inhale and exhale quickly and forcefully for 10 seconds.

\section{Respiratory muscle strength test}

Respiratory muscle strength was assessed by measuring the MIP and MEP in a seated position using a portable handheld mouth pressure meter (i.e., MicroRPM) with a nose clip. For the MIP measurement, participants were asked to exhale until they felt no air remaining in their lungs (starting with the functional residual capacity point). They then held the device on their mouth and inhaled forcefully for 1-2 seconds. For the MEP measurement, participants were asked to inhale until their lungs were completely filled with air (starting with the total lung capacity [TLC] point). They then kept the device on their mouth and exhaled forcefully for 1-2 seconds. 


\section{Aerobic capacity evaluation}

Aerobic capacity was assessed using the 6-MWD following the guidelines of the 2002 American Thoracic Society. A modified $25 \mathrm{~m}$ straight walking test with turning points was performed on the 4th floor next to the Critical Pulmonary Division. Participants were asked to wear comfortable clothes and shoes during the test. Their vital signs (e.g., resting heart rate and blood pressure) were evaluated before and after the test. Participants were asked to walk at a comfortable pace for 6 minutes under the supervision of a nurse. They were permitted to terminate and rest during the test if they sensed heavy dyspnea. Researchers collected the data and calculated the maximum oxygen consumption $\left(\mathrm{VO}_{2} \mathrm{max}\right)$ using the following formula:

$\mathrm{VO}_{2} \max (\mathrm{mL} / \mathrm{kg} / \mathrm{min})=[0.02 \times$ distance $(\mathrm{m})]-[0.191 \times$ age (year) $]-[0.07 \times$ weight $(\mathrm{kg})]+[0.09 \times$ height $(\mathrm{cm})]+[0.26 \times$ heart rate $(\mathrm{bpm})] \times$ systolic blood pressure $(\mathrm{mm} \mathrm{Hg}) \times\left(10^{-3}\right)+245$.

\section{Effects of exercise on COPD symptoms}

Effects of exercise on COPD symptoms were assessed using mMRC and CAT scores. The mMRC is a questionnaire used to evaluate the level of disability in the daily life of patients with $\mathrm{COPD}^{27}$. The mMRC has a 5-point $(0-4)$ scale based on the severity of dyspnea ${ }^{28}$, whereas the CAT is a patient-completed instrument to assess and quantify the quality of life and burden of symptoms in patients with COPD. It consists of eight questions, each of which has a semantic 6-point $(0-5)$ differential scale, providing a total score of up to 40 points. Scores 0-10, 11-20, 21-30, and 31-40 represented mild, moderate, severe, and very severe clinical impact, respectively ${ }^{29}$.

\section{Statistical analysis}

All data were statistically analyzed for their normal distribution using the Shapiro-Wilk test before calculating the mean and standard deviation (SD). Characteristic data, pulmonary function, respiratory muscle strength, inflammatory cytokines, oxidative stress, aerobic capacity, and effects on COPD symptoms were analyzed using independent t-tests to compare groups. A paired t-test was used to compare results before and after breathing exercises. All statistical analyses were performed using the SPSS software program version 17.0 (SPSS Inc., Chicago, IL, USA) for Windows. Tests were considered to be significant when p-value was less than 0.05 .

\section{Results}

Eligible participants were allocated into two groups (Figure 1): $\mathrm{DB}(\mathrm{n}=9)$ and FB $(\mathrm{n}=10)$. Three participants dropped out of this study because of scheduling difficulties. Due to the coronavirus disease 2019 pandemic, hospital visits were limited and patients were concerned. Therefore, DB and FB groups each had 8 patients ( 7 males and 1 female in each group). Medication information of participants was identical for both groups. The majority of them had COPD stage II. They were given a bronchodilator inhaler.

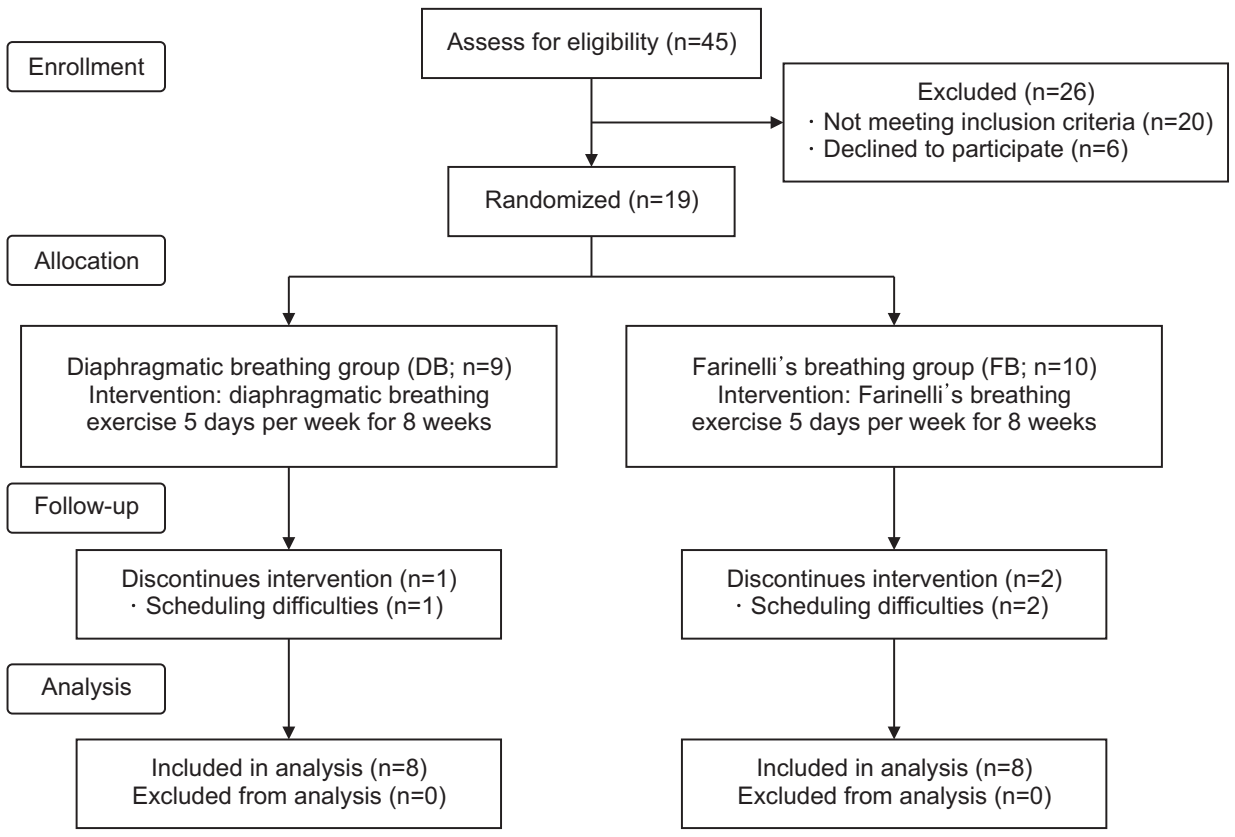

Figure 1. CONSORT 2010 flow diagram showing participant allocation, followup, and analysis. 
Table 1. Physiological characteristics of subjects in DB and FB groups

\begin{tabular}{|c|c|c|c|}
\hline Variable & DB $(n=8)$ & FB $(n=8)$ & p-value \\
\hline Age, yr & $66.63 \pm 9$ & $67.63 \pm 7.93$ & 0.82 \\
\hline Male:Female & $7: 1$ & $7: 1$ & - \\
\hline Weight, kg & $70.78 \pm 15.62$ & $70.65 \pm 14.02$ & 0.99 \\
\hline RHR, bpm & $83.75 \pm 20.23$ & $86.00 \pm 16.13$ & 0.80 \\
\hline $\mathrm{BMI}, \mathrm{kg} / \mathrm{m}^{2}$ & $25.86 \pm 5.99$ & $26.31 \pm 4.21$ & 0.86 \\
\hline SBP, mm Hg & $116.38 \pm 23.31$ & $127.88 \pm 4.57$ & 0.19 \\
\hline DBP, mm Hg & $73.50 \pm 10.98$ & $80.75 \pm 4.52$ & 0.10 \\
\hline PBF, $\%$ & $29.69 \pm 8.15$ & $30.35 \pm 7.91$ & 0.92 \\
\hline $\mathrm{SpO}_{2}, \%$ & $96.63 \pm 1.69$ & $95.25 \pm 1.98$ & 0.16 \\
\hline FVC, L & $2.47 \pm 0.49$ & $2.34 \pm 0.69$ & 0.68 \\
\hline $\mathrm{FEV}_{1}$ predicted, \% & $73.36 \pm 8.81$ & $72.01 \pm 11.37$ & 0.80 \\
\hline FVC predicted, \% & $78.38 \pm 17.80$ & $74.25 \pm 20.82$ & 0.68 \\
\hline
\end{tabular}

Values are presented as mean \pm SD.

DB: diaphragmatic breathing; FB: Farinelli's breathing; RHR: resting heart rate; BMI: body mass index; SBP: systolic blood pressure; DBP: diastolic blood pressure; $\mathrm{PBF}$ : percent of body fat; $\mathrm{SpO}_{2}$ : oxygen saturation; $\mathrm{FVC}$ : forced vital capacity; $\mathrm{FEV}_{1}$ : forced expiratory volume in 1 second.
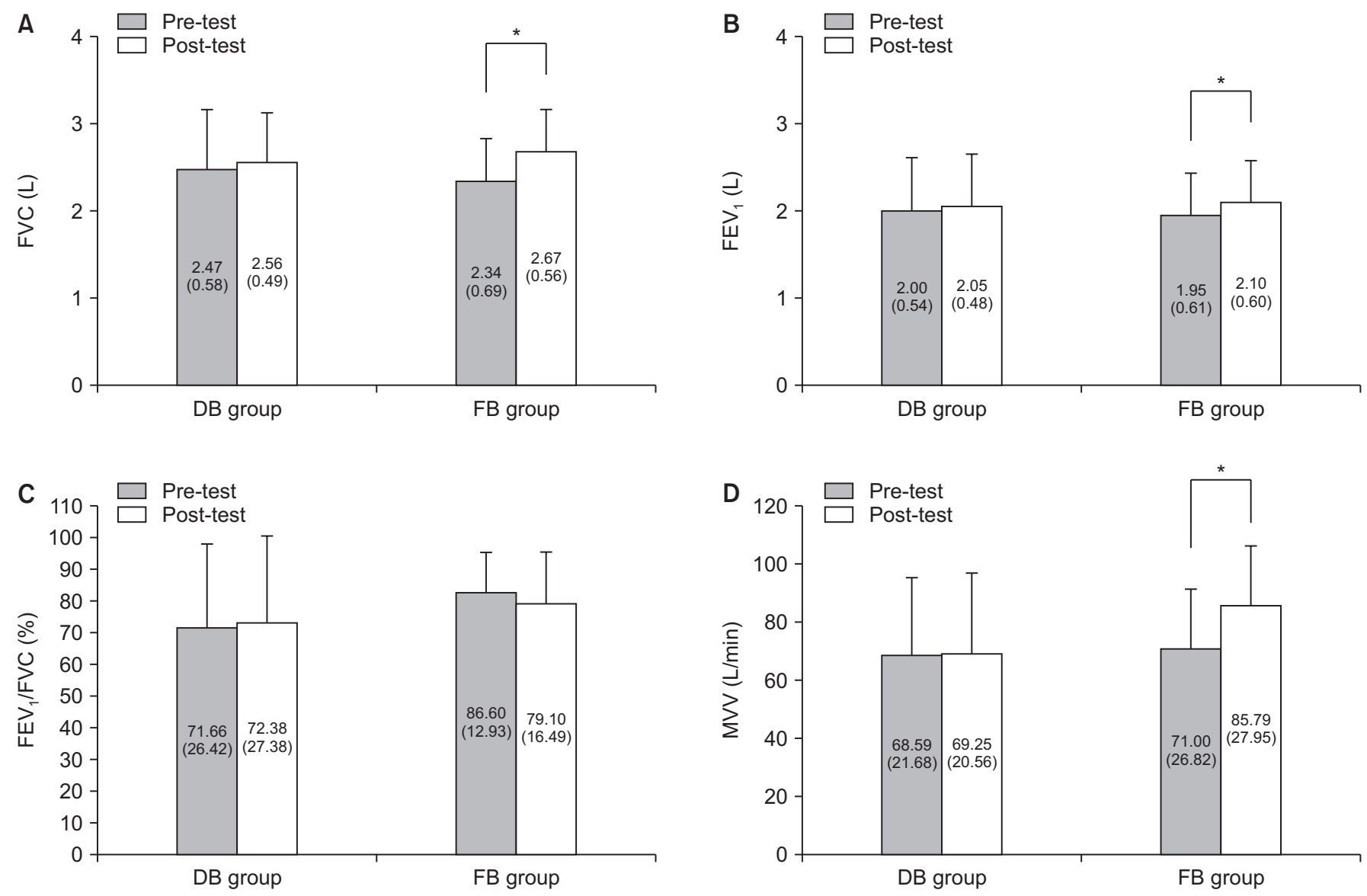

Figure 2. (A-D) Comparisons of pulmonary function between pre- and post-breathing exercises and between DB and FB groups. Values are presented as mean \pm SD. * $<<0.05$ vs. Pre-test. DB: diaphragmatic breathing; FB: Farinelli breathing; SD: standard deviation; FVC: forced vital capacity; $\mathrm{FEV}_{1}$ : forced expiratory volume in 1 second; $\mathrm{MVV}$ : maximal voluntary. 


\section{Physiological characteristic data}

No variable was significantly different at baseline between the two groups (all $\mathrm{p}>0.05$ ). Data are expressed as mean and SD (Table 1).

\section{Pulmonary function}

The FB group showed significant changes in FVC $(\mathrm{p}=0.026)$, $\mathrm{FEV}_{1}(\mathrm{p}=0.012)$, and MVV maneuver $(\mathrm{p}<0.001)$ after the exercise. However, there was no significant difference in pulmonary function between the two groups (Figure 2).

\section{Respiratory muscle strength and aerobic capacity}

In the DB group, only MEP was changed significantly $(\mathrm{p}=0.013)$ after the exercise. In the FB group, both MIP $(\mathrm{p}<0.001)$ and MEP $(\mathrm{p}<0.001)$ were changed significantly. Both MIP and MEP were significantly higher in the FB group than those in the DB group when they were compared at posttest $(\mathrm{p}=0.016$ and $\mathrm{p}=0.039$, respectively). Moreover, after 8 weeks of breathing exercises, $6-\mathrm{MWD}(\mathrm{p}=0.005$ and $\mathrm{p}=0.002$, respectively) and $\mathrm{VO}_{2} \max (\mathrm{p}=0.010$ and $\mathrm{p}=0.035$, respectively) were increased significantly in DB and FB groups when compared to those at pre-test, although they were not significantly different between the two groups (Figure 3).
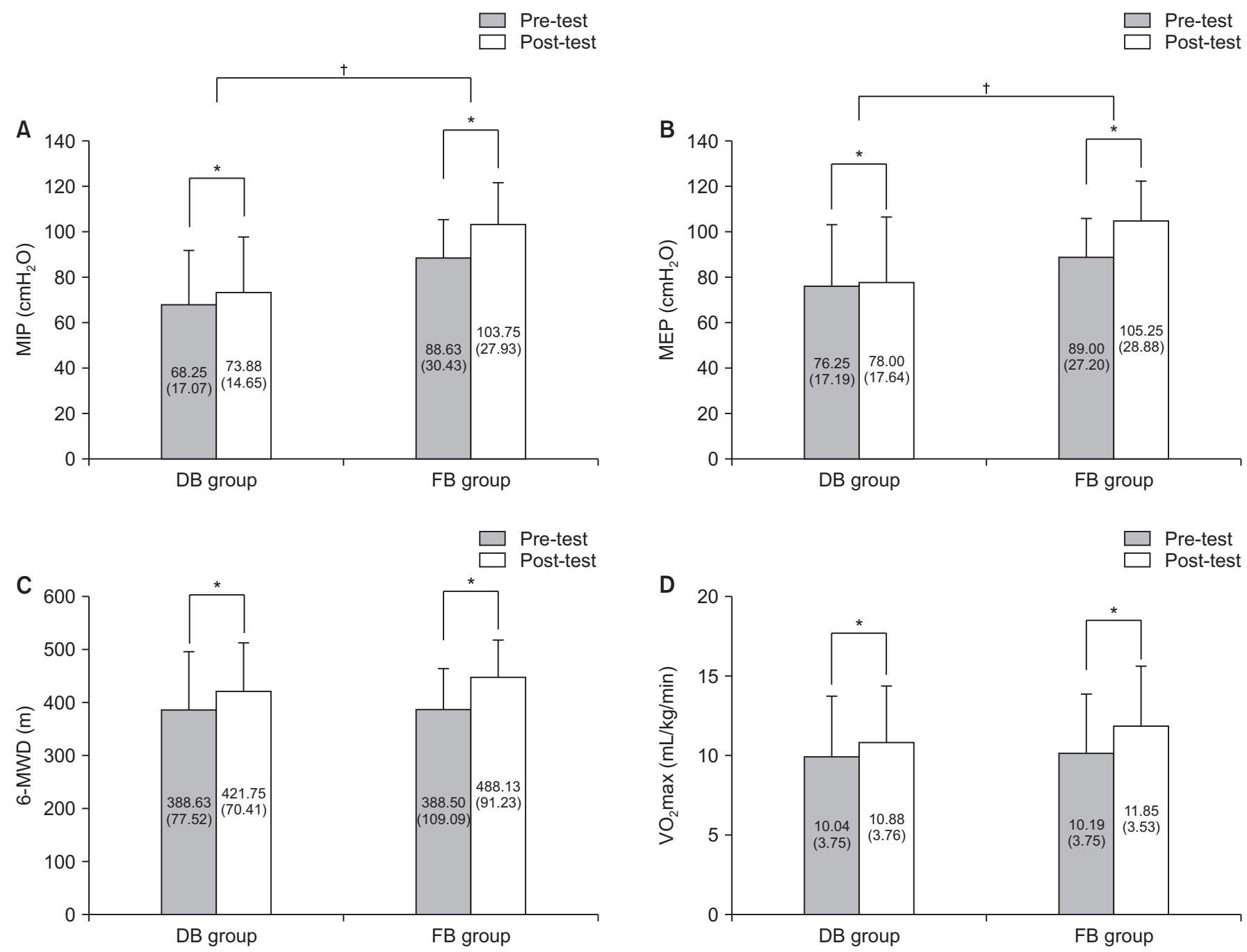

Figure 3. (A-D) Comparisons of respiratory muscle strength and aerobic capacity between pre- and post-breathing exercise and between DB and FB groups. Values are presented as mean \pm SD. ${ }^{*} \mathrm{p}<0.05$ vs. Pre-test. ${ }^{\dagger} \mathrm{p}<0.05$ vs. DB group. DB: diaphragmatic breathing; FB: Farinelli breathing; SD: standard deviation; MIP: maximal inspiratory pressure; MEP: maximal expiratory pressure; 6-MWD: 6-minute walk distance; $\mathrm{VO}_{2}$ max: maximal oxygen consumption. 


\section{Inflammatory cytokine and oxidative stress}

There was no statistical change in inflammatory cytokine or oxidative stress in the DB group after the exercise. However, TNF- $\alpha(\mathrm{p}=0.027)$ and malondialdehyde (MDA) levels $(p=0.015)$ were significantly reduced in the FB group after the exercise, although they were not significantly different between the two groups at post-test (Figure 4).

\section{Effects on COPD symptoms}

Regarding effects of exercise on COPD symptoms, both groups showed significantly reduced CAT scores $(\mathrm{p}=0.017$ and $p=0.005$ ), although there was no significant difference in any symptom score between the two groups at post-test (Figure 5).

\section{Discussion}

The main finding of this study was that respiratory functions and COPD symptoms in patients with stable (stage I and
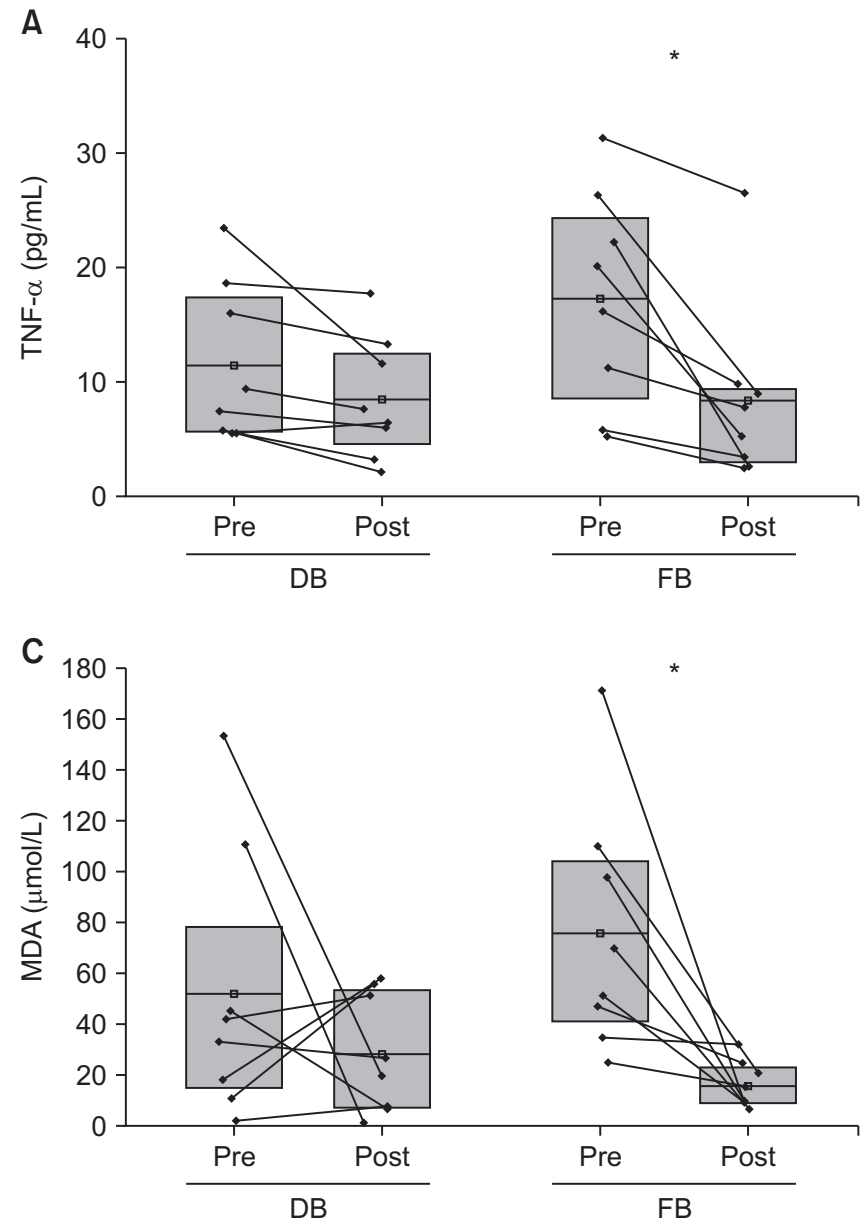

stage II) COPD were improved, which was a novel finding. Respiratory functions and dyspnea symptoms are known to worsen in patients with COPD. Two main pathophysiological characteristics, chronic bronchitis and emphysema, can lead to excessive sputum production and narrowing of airways, resulting in an insufficient capacity for gas exchange $\mathrm{e}^{30}$. Moreover, patients with COPD have hyperinflation-induced respiratory muscle dysfunction, limited tidal volume, and limited inspiratory reserve volume ${ }^{30}$, resulting in an abnormal breathing pattern ${ }^{31}$. COPD patients in the FB group had significantly higher MIP and MEP than those in the DB group, emphasizing the activation of the diaphragm. The suspension phase is similar to the sustained maximal inspiration breathing technique, where the inhalation forms the FRC until the TLC has been reached in order to increase the alveolar distension. Its use can result in lung stretching and increased $\mathrm{VC}^{32}$. Additionally, the exhalation phase resembles the pursedlip breathing technique, where the patient exhales through pursed lips, resulting in reduced gas trapping ${ }^{33}$. By performing the FB exercise, participants breathed in slowly to activate the diaphragm muscle while in the suspension phase until achieving the struggle phase. This increased the magnitude and

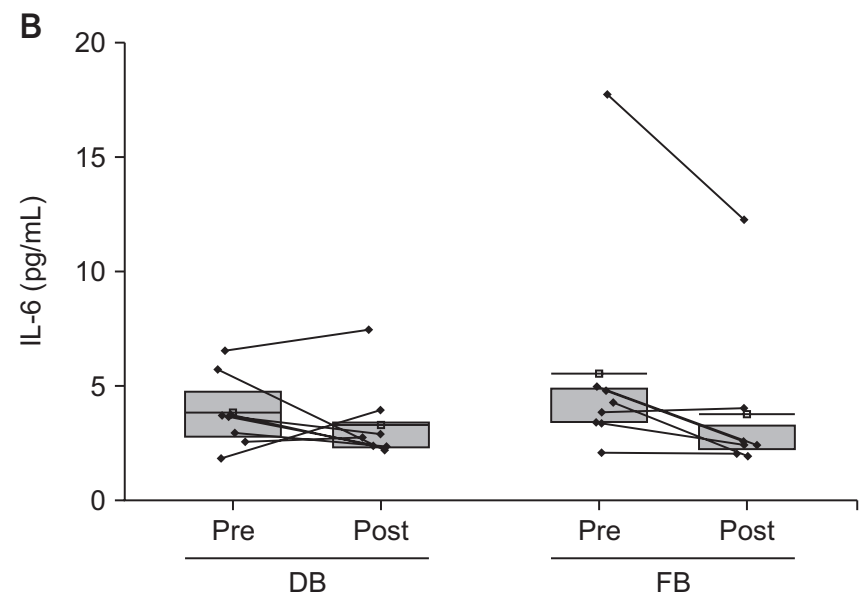

Figure 4. Comparisions of inflammatory cytokine (A, TNF- $\alpha$ and B, IL-6) and oxidative stress (MDA) (C) levels between pre- and postbreathing exercise and between DB and FB groups. * $\mathrm{p}<0.05$ vs. Pretest. DB: diaphragmatic breathing; FB: Farinelli breathing; TNF- $\alpha$ : tumor necrosis factor- $\alpha$; IL-6: interleukin-6; MDA: malondialdehyde. 

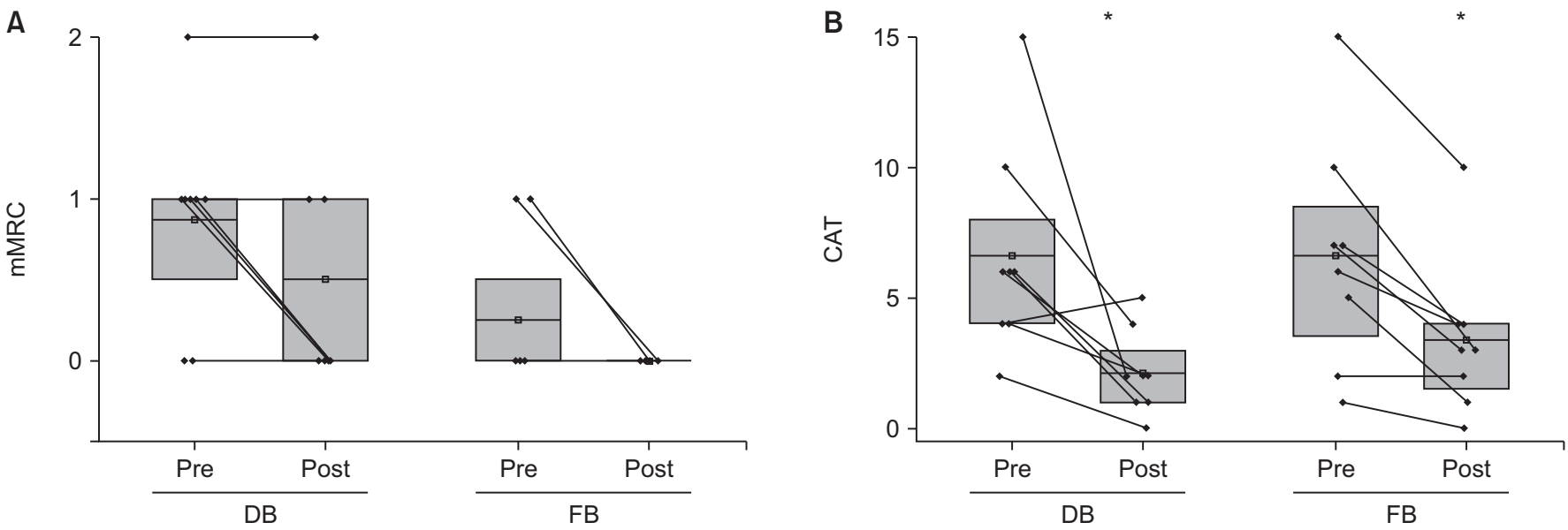

Figure 5. Comparisions of effects of exercise on COPD symptoms (A, mMRC and B, CAT) between pre- and post-breathing exercise and between DB and FB groups. ${ }^{*} \mathrm{p}<0.05$ vs. Pre-test. COPD: chronic obstructive pulmonary disease; mMRC: modified Medical Research Council; CAT: COPD assessment test; DB: diaphragmatic breathing; FB: Farinelli breathing.

frequency of respiratory muscle contraction, resulting in an enhanced gaseous exchange efficiency and improved respiratory muscle strength represented by increased MIP and MEP. Moreover, this breathing exercise increased the MVV maneuver known to represent respiratory muscle endurance ${ }^{34}$. This suggests that the FB exercise can improve respiratory function in patients with COPD.

The better the respiratory function of COPD patients, the greater the health benefits. Immune responses play an important role in respiratory function. ROS can change biological molecules, signaling pathways, and activities of antioxidant molecules. Elevated cytokines and oxidative stress are known to be implicated in the pathogenesis of COPD. The repetitive breathing cycle of FB can result in an alteration in the immune response of respiratory muscles by downregulating inflammatory cytokines and decreasing TNF- $\alpha$ production from macrophages ${ }^{35}$. This mechanism not only decreases TNF- $\alpha$ level, but also reduces ROS generation, which may result in decreased MDA levels. These findings indicate that FB exercise can increase respiratory muscle strength, minimize air trapping, and decrease TNF- $\alpha$ and MDA levels, which can affect pulmonary fibrosis and emphysema, leading to decreased pulmonary obstruction, improved $\mathrm{FEV}_{1}$, and reduced cardinal symptoms.

Dyspnea is an important respiratory symptom present in COPD. It is caused by decreased $\mathrm{O}_{2}$ levels in the aorta. Increased $\mathrm{H}^{+}$and $\mathrm{CO}_{2}$ levels in arteries can cause respiratory discomfort and breathlessness, triggering the medulla oblongata to initiate inspiratory neural drive in lung receptors and respiratory muscles to instruct breathing ${ }^{36}$, where breathing exercises enable efficient gas exchange. Reduced air retention in the lungs can modify the efficacy of breathing patterns to reduce dyspnea ${ }^{23,34,37}$. Our results indicated that the FB group had decreased CAT scores after 8 weeks of breathing exercise sessions and improved ventilatory functions, dyspnea, health status, and physical activity in patients with COPD. Decreased CAT scores after treatment indicate that FB can reduce dyspnea and general symptom perception in patients with COPD.

The aim of the study was to compare effects of FB and DB exercises on respiratory function and symptoms in patients with COPD. The following limitations of this study should be considered when interpreting results of this study. First, the number of enrolled participants was small. Future research enrolling more patients with COPD having varying severity is needed to validate possible applications of these findings in patients using combined medication and perform comparisons with other breathing exercises. Second, we discovered that patients with COPD were overweight (the average BMI of participants was above $25 \mathrm{~kg} / \mathrm{m}^{2}$ ) with decent lung function, different from prior studies ${ }^{38,39}$. The reason for such finding might be because patients in this study had COPD stages I (mild) and II (moderate). Their appetite and dyspnea might not be severe enough to cause weight loss or decrease their lung functions. Thus, recruiting more patients at various stages of COPD is needed in the future.

In conclusion, for COPD patients, the FB exercise can open up airways sufficiently enough to allow more air to pass through and aid in the control of shortness of breath. FB exercise is a quick and easy technique that can reduce a patient's breathing rate, making each breath more efficient. Although this breathing technique is somewhat complex, if practiced on a regular basis, it may become habitual. This exercise can enhance pulmonary function, respiratory muscle strength, aerobic capacity, and reduce inflammatory cytokines, oxidative stress, and COPD symptoms. Healthcare practitioners such as nurses, physicians, and exercise therapists play important roles in promoting this beneficial exercise as a useful alternative for COPD patients by incorporating it into their advice, 
patient manuals, and practical guidelines for improving health status and quality of life.

\section{Authors' Contributions}

Conceptualization: Ittinirundorn S, Wongsaita N, Tongtako W. Methodology: Ittinirundorn S, Wongsaita N. Formal analysis: Ittinirundorn S, Wongsaita N, Tongtako W. Data curation: Ittinirundorn S, Wongsaita N, Somboonviboon D, Tongtako W. Writing - original draft preparation: Ittinirundorn S. Writing review and editing: Tongtako W. Approval of final manuscript: all authors.

\section{Conflicts of Interest}

No potential conflict of interest relevant to this article was reported.

\section{Acknowledgments}

The authors thank all participants and research assistants for helping and organizing this study. The authors also thank all nurses in Specialty Cline Pulmonary-Allergy \& Immunology, Phramongkutklao hospital, Bangkok who provided a place for collecting data and Supranee Buranapraditkun, Ph.D. for cytokines and oxidative stress analysis.

\section{Funding}

This research was supported by the 90th Anniversary Chulalongkorn University Fund (Ratchadaphiseksomphot Endowment Fund) of Chulalongkorn University and Faculty of Sports Science Fund of Chulalongkorn University.

\section{References}

1. Brandsma CA, Van den Berge M, Hackett TL, Brusselle G, Timens W. Recent advances in chronic obstructive pulmonary disease pathogenesis: from disease mechanisms to precision medicine. J Pathol 2020;250:624-35.

2. Mathioudakis AG, Vanfleteren L, Lahousse L, Higham A, Allinson JP, Gotera C, et al. Current developments and future directions in COPD. Eur Respir Rev 2020;29:200289.

3. Orozco-Levi M. Structure and function of the respiratory muscles in patients with COPD: impairment or adaptation? Eur Respir J Suppl 2003;46:41s-51s.

4. Anzueto A, Miravitlles M. Pathophysiology of dyspnea in COPD. Postgrad Med 2017;129:366-74.
5. Rahman I. Oxidative stress in pathogenesis of chronic obstructive pulmonary disease: cellular and molecular mechanisms. Cell Biochem Biophys 2005;43:167-88.

6. Domej W, Oettl K, Renner W. Oxidative stress and free radicals in COPD: implications and relevance for treatment. Int J Chron Obstruct Pulmon Dis 2014;9:1207-24.

7. Leelarungrayub J, Puntumetakul R, Sriboonreung T, Pothasak Y, Klaphajone J. Preliminary study: comparative effects of lung volume therapy between slow and fast deep-breathing techniques on pulmonary function, respiratory muscle strength, oxidative stress, cytokines, 6-minute walking distance, and quality of life in persons with COPD. Int J Chron Obstruct Pulmon Dis 2018;13:3909-21.

8. Kirkham PA, Barnes PJ. Oxidative stress in COPD. Chest 2013;144:266-73.

9. Ahmad A, Shameem M, Husain Q. Altered oxidant-antioxidant levels in the disease prognosis of chronic obstructive pulmonary disease. Int J Tuberc Lung Dis 2013;17:1104-9.

10. Nield MA, Soo Hoo GW, Roper JM, Santiago S. Efficacy of pursed-lips breathing: a breathing pattern retraining strategy for dyspnea reduction. J Cardiopulm Rehabil Prev 2007;27:237-44.

11. Yamaguti WP, Claudino RC, Neto AP, Chammas MC, Gomes AC, Salge JM, et al. Diaphragmatic breathing training program improves abdominal motion during natural breathing in patients with chronic obstructive pulmonary disease: a randomized controlled trial. Arch Phys Med Rehabil 2012;93:571-7.

12. Jansang S, Mickleborough T, Suksom D. Effects of pursed-lip breathing exercise using windmill toy on lung function and respiratory muscle strength in the elderly. J Med Assoc Thai 2016;99:1046-51.

13. Lu Y, Li P, Li N, Wang Z, Li J, Liu X, et al. Effects of home-based breathing exercises in subjects with COPD. Respir Care 2020; 65:377-87.

14. Ubolnuar N, Tantisuwat A, Thaveeratitham P, Lertmaharit S, Kruapanich C, Mathiyakom W. Effects of breathing exercises in patients with chronic obstructive pulmonary disease: systematic review and meta-analysis. Ann Rehabil Med 2019;43: 509-23.

15. Hill CJ, Lazzeri M, D’Abrosca F. Breathing exercises and mucus clearance techniques in pulmonary rehabilitation. In: Clini E, Holland AE, Pitta F, Troosters T, editors. Textbook of pulmonary rehabilitation. Cham: Springer International Publishing; 2018. p. 205-16.

16. Roy AS, Bandyopadhyay A. Pulmonary function studies in female singers of Kolkata, India. J Hum Ergol (Tokyo) 2015;44: 75-81.

17. Imam MI JJ, Akor-Dewu MB. A cross sectional study on lung functions in athletes, singers, and individuals with sedentary lifestyles in Ahmadu Bello University, Zaria. Bayero J Pure Appl Sci 2017;10:83-7.

18. Seo K, Park SH, Park K. Effects of diaphragm respiration exercise on pulmonary function of male smokers in their twen- 
ties. J Phys Ther Sci 2015;27:2313-5.

19. Visser FJ, Ramlal S, Dekhuijzen PN, Heijdra YF. Pursed-lips breathing improves inspiratory capacity in chronic obstructive pulmonary disease. Respiration 2011;81:372-8.

20. Zhang ZQ, Chen RC, Yang QK, Li P, Wang CZ, Zhang ZH. A randomized controlled trial study of pulmonary rehabilitation with respiratory physiology as the guide on prognosis in patients with chronic obstructive pulmonary disease. Zhongguo Wei Zhong Bing Ji Jiu Yi Xue 2008;20:607-10.

21. Salomoni S, van den Hoorn W, Hodges P. Breathing and singing: objective characterization of breathing patterns in classical singers. PLoS One 2016;11:e0155084.

22. Gosselink R. Breathing techniques in patients with chronic obstructive pulmonary disease (COPD). Chron Respir Dis 2004;1:163-72.

23. Tamplin J, Baker FA, Grocke D, Brazzale DJ, Pretto JJ, Ruehland WR, et al. Effect of singing on respiratory function, voice, and mood after quadriplegia: a randomized controlled trial. Arch Phys Med Rehabil 2013;94:426-34.

24. Cancelliero-Gaiad KM, Ike D, Pantoni CB, Borghi-Silva A, Costa D. Respiratory pattern of diaphragmatic breathing and pilates breathing in COPD subjects. Braz J Phys Ther 2014;18:291-9.

25. Afrodite E, Eirini G, Emmanouil S, Aikaterini H, The effect of diaphragmatic breathing on dyspnea and exercise tolerance during exercise in COPD patients. Chest 2015;148(Suppl): 704A.

26. Budiman, Garnewi S. Effects of diaphragmatic breathing exercise on the degree of breathlessness in patients with chronic obstructive pulmonary disease. Iran Rehabil J 2021;19:69-74.

27. Kovelis D, Segretti NO, Probst VS, Lareau SC, Brunetto AF, Pitta F. Validation of the modified pulmonary functional status and dyspnea questionnaire and the Medical Research Council scale for use in Brazilian patients with chronic obstructive pulmonary disease. J Bras Pneumol 2008;34:1008-18.

28. Bestall JC, Paul EA, Garrod R, Garnham R, Jones PW, Wedzicha JA. Usefulness of the Medical Research Council (MRC) dyspnoea scale as a measure of disability in patients with chronic obstructive pulmonary disease. Thorax 1999;54:5816.

29. Cheng SL, Lin CH, Wang CC, Chan MC, Hsu JY, Hang LW, et al. Comparison between COPD Assessment Test (CAT) and modified Medical Research Council (mMRC) dyspnea scores for evaluation of clinical symptoms, comorbidities and medical resources utilization in COPD patients. J Formos Med Assoc 2019;118:429-35.

30. Suh MR, Kim DH, Jung J, Kim B, Lee JW, Choi WA, et al. Clinical implication of maximal voluntary ventilation in myotonic muscular dystrophy. Medicine (Baltimore) 2019;98:e15321.

31. Rabe KF, Hurd S, Anzueto A, Barnes PJ, Buist SA, Calverley P, et al. Global strategy for the diagnosis, management, and prevention of chronic obstructive pulmonary disease: GOLD executive summary. Am J Respir Crit Care Med 2007;176:53255.

32. Garrod R, Marshall J, Barley E, Fredericks S, Hagan G. The relationship between inflammatory markers and disability in chronic obstructive pulmonary disease (COPD). Prim Care Respir J 2007;16:236-40.

33. Cross TJ, Breskovic T, Sabapathy S, Zubin Maslov P, Johnson BD, Dujic Z. Respiratory muscle pressure development during breath holding in apnea divers. Med Sci Sports Exerc 2013;45:93-101.

34. Mendes LP, Moraes KS, Hoffman M, Vieira DS, Ribeiro-Samora GA, Lage SM, et al. Effects of diaphragmatic breathing with and without pursed-lips breathing in subjects with COPD. Respir Care 2019;64:136-44.

35. Beavers KM, Brinkley TE, Nicklas BJ. Effect of exercise training on chronic inflammation. Clin Chim Acta 2010;411:78593.

36. Hanania NA, O'Donnell DE. Activity-related dyspnea in chronic obstructive pulmonary disease: physical and psychological consequences, unmet needs, and future directions. Int J Chron Obstruct Pulmon Dis 2019;14:1127-38.

37. Fernandes M, Cukier A, Feltrim MI. Efficacy of diaphragmatic breathing in patients with chronic obstructive pulmonary disease. Chron Respir Dis 2011;8:237-44.

38. Prescott E, Almdal T, Mikkelsen KL, Tofteng CL, Vestbo J, Lange P. Prognostic value of weight change in chronic obstructive pulmonary disease: results from the Copenhagen City Heart Study. Eur Respir J 2002;20:539-44.

39. McDonald V, Gibson P, Scott H, Baines P, Hensley M, Pretto J, et al. Obesity in COPD, how should it be managed?: the effect of weight loss and resistance training in obese COPD patients. Eur Respir J 2014;44:P3035. 